\title{
Efeitos da terapia fonoaudiológica associada à eletroestimulação neuromuscular nas funções estomatognáticas e expressão facial na Síndrome de Moebius: relato de experiência
}

\author{
Effects of speech therapy associated with neuromuscular electrical stimulation on stomatognathic
}

functions and facial expression in Moebius Syndrome: an experience report

Efectos de la terapia del habla asociada con la estimulación eléctrica neuromuscular sobre las

funciones estomatognáticas y la expresión facial en el Síndrome de Moebius: un informe de experiencia

\section{Resumo}

A Síndrome de Moebius (SM) é uma doença rara, que acomete cerca de 1 indivíduo a cada 250 mil cidadãos. Sistematizada inicialmente por Paul Moebius, trata-se de uma síndrome que acarreta a paralisia total ou parcial do nervo facial, podendo acrescer ainda a paralisia uni ou bilateral. Sabe-se que a fonoaudiologia busca propor estratégias para o pleno desenvolvimento do indivíduo, para isso, buscou-se a partir da metodologia de relato de caso, evidenciar a evolução de uma paciente diagnosticada com SM, submetida a um protocolo especial de fonoterapia convencional associada a estimulação elétrica (FES), durante o período de março a junho de 2021. Pode-se perceber, que embora seja um tema que carece de maiores e mais afunilados estudos, posto a escassez de material encontrado para análise, a fonoterapia associada ao FES demonstrou-se positiva no quadro evolutivo da paciente, sobretudo nas funções de mastigação, deglutição e praxias orofaciais. Por meio da análise também foi possível notar que as contribuições da intervenção interdisciplinar entre a equipe profissional e família também contribui positivamente, sendo de extrema importância no momento da terapia.

Palavras-chave: Fonoterapia convencional; Corrente FES; Exercícios fonoterápicos.

\begin{abstract}
Moebius Syndrome (MS) is a rare disease that affects about 1 individual in every 250 thousand citizens. Coined by Paul Moebius, it is a syndrome that leads to total or partial paralysis of the facial nerve, which can also be accompanied by unilateral or bilateral paralysis. Thus, it is known that speech therapy seeks to propose strategies for the full development of the individual, for this, it was sought, from the case report methodology, to evidence the evolution of a patient diagnosed with MS, submitted to a special protocol of conventional speech therapy associated with electrical stimulation (FES), during the period from March to June 2021. It can be seen that, although it is a topic that needs further and more detailed studies, given the scarcity of material found for analysis, speech therapy associated with FES, it was positive in the patient's evolution, especially in the areas of chewing, swallowing and
\end{abstract}


orofacial praxis. Through the analysis, it was also possible to note that the contributions of interdisciplinary work between the patient's family and the professional team also contribute positively, being extremely important at the time of therapy.

Keywords: Conventional speech therapy; Electro-stimulation; Moebius syndrome.

\section{Resumen}

El Síndrome de Moebius (EM) es una enfermedad rara que afecta aproximadamente a 1 persona de cada 250 mil ciudadanos. Acuñado por Paul Moebius, es un síndrome que conduce a la parálisis total o parcial del nervio, que también puede ir acompañada de parálisis facial unilateral o bilateral. Así, se sabe que la logopedia busca proponer el desarrollo pleno del individuo, para ello se buscó, a partir de la metodología de relato de caso, evidenciar la evolución de un paciente con EM, sometido a un protocolo especial de logopedia convencional. asociado a la estimulación eléctrica (FES), durante el período de marzo a junio de 2021. Se puede observar que, si bien es un tema que necesita estudios más detallados, dada la escasez de material encontrado para el análisis, la fonoaudiología asociada a FES, fue positivo en la evolución del paciente, especialmente en las áreas de masticación, deglución y praxis orofacial. A través del análisis, también se pudo constatar que los aportes del trabajo interdisciplinario entre la familia del paciente y el equipo profesional también contribuyen positivamente, siendo sumamente importante a la hora de la terapia.

Palabras clave: Terapia del habla convencional; Electroestimulación; Síndrome de Moebius.

\section{Introdução}

Foi de Von Graaefe o primeiro entendimento acerca da diplegia facial congênita, sendo que este, em 1880, descreveu o caso de uma paciente com paralisia do VII par craniano. Logo em 1888, e após, em 1982, Moebius verificou alguns casos do envolvimento congênito abducente e facial, explicitando que estas anomalias trataram de entidades independentes (Kumar, 1990; Pupo Filho, 1999; Miller \& Stromland, 1999). Por volta do ano de 1982, Paul Moebius, um conceituado neurologista da Alemanha, conseguiu compilar 43 casos de paralisia facial congênita e adquirida, descrevendo-os e analisando-os. A partir deste fato histórico, emerge o entendimento da Síndrome de Moebius (SM), que na atualidade também é intitulada como aplasia nuclear, paralisia oculofacial congênita ou diplegia facial (Diogo et al., 2009).

A síndrome ou sequência de Moebius (SM) é caracterizada pela paralisia congênita parcial ou completa do nervo facial, associada a pares cranianos e sobretudo ao nervo abducente (Kumar, 1990; Lammens et al., 1998). O nervo facial é paralisado, por sua estrutura não apresentar constância no trajeto. O nervo facial é formado pelo nervo motor - que inerva os músculos da face, além do intermediário de Wrisberg, que possui fibras sensitivas e parassimpáticas (Freitas et al., 2006).

Conforme assevera Freitas et al. (2006, p. 2) a SM pode ser acompanhada por paralisia unilateral ou bilateral de outros nervos cranianos, com destaque para o nervo abducente, e menor frequência no oculomotor, troclear, glossofaríngeo, vago e do hipoglosso, determinando distúrbios de sensibilidade nas regiões inervadas pelo trigêmeo, disfagia, disfonia e paralisia do músculo reto lateral, verificados em diferentes combinações.

Para Picciolini et al. (2016) a prevalência estimada da SM é de 1 para 250 mil nascidos vivos, com prevalência em ambos os sexos, sendo que a maioria dos casos é esporádico, contudo, nos casos em que há uma predisponência familiar para o transtorno, há uma estimativa de $2 \%$. De todo modo, estudos indicam que a incidência da SM, pode ser relacionada à utilização ilegal do fármaco Misoprostol, que é análogo à Prostaglandina, comumente utilizado como abortivo no primeiro trimestre da gestação. Para Larrandaburu (1990, p. 1) “a síndrome parece estar relacionada com a insuficiência útero-placentária em determinado momento da organogênese, levando à agenesia dos núcleos dos pares cranianos no tronco cerebral, lesões supranucleares e pós-nucleares”.

A comunidade acadêmica das ciências da saúde busca constantemente atualizar seus conhecimentos a respeito da utilização de instrumentos e possibilidades que viabilizem a recuperação dos pacientes, nos mais diversos casos, principalmente no que tange aos aparatos que "atuam na musculatura e inervação do corpo visando aplicação clínica" (Romansina et al., 2020, p. 2). Além disso, uma das correntes mais utilizadas nestas áreas é a Eletroestimulação Elétrica Funcional (FES), que se liga especialmente ao sistema eferente dos indivíduos, cuja corrente é conduzida por meio de 
eletrodos transcutâneos, explorando um resultado ou ação diante da membrana excitável, ao promover uma contração muscular, contudo, é válido ressaltar que tal estímulo está ligado especialmente à frequência, duração e outros aspectos da aplicação da corrente.

Estudos de Romansina et al. (2020) e Pinheiro et al., (2018), corroboram com o posicionamento de que a corrente FES é uma grande aliada aos tratamentos fonoaudiológicos, uma vez que contribui com o fortalecimento dos músculos, ampliação da resistência e do fluxo sanguíneo na região que está recebendo os respectivos estímulos, embora seja alvo de pesquisas recentes e poucas conclusões para a comunidade.

Nesta perspectiva, objetivou-se, a partir do presente relato de experiência, expor as contribuições da eletroestimulação associada à fonoterapia convencional no desenvolvimento das funções estomatognáticas e expressão facial de uma paciente portadora da SM, bem como explicitar as contribuições do trabalho multidisciplinar da família e do profissional fonoaudiólogo, para a eficácia do tratamento.

\section{Metodologia}

Trata-se de um relato de experiência, baseado em um caso de uma paciente diagnosticada com a SM, de 6 anos, sexo feminino, atendida no Centro de Atenção e Pesquisa em Anomalias Craniofaciais (CEAPAC), no Hospital Universitário do Oeste do Paraná (HUOP) em Cascavel-PR, no período correspondente aos meses de março a junho do ano de 2021. Para a viabilidade do estudo, este foi dividido em quatro etapas.

Sendo assim, para a participação no projeto de pesquisa, os responsáveis assinaram o Termo de Consentimento Livre e Esclarecimento (TCLE) que faz parte do projeto intitulado "Anomalias Craniofaciais: o tratamento sob a ótica multidisciplinar" com aprovação do comitê de ética em pesquisa com seres humanos do HUOP, sob o parecer de número 4.250.143.

A primeira etapa consistiu na realização da anamnese, cujos responsáveis pela criança responderam a perguntas objetivas, acerca das principais queixas, histórico, condições de saúde, queixas específicas quanto à fala, estética facial, respiração, alimentação, audição, voz e escolaridade.

Na segunda etapa, a paciente foi avaliada por meio de protocolo de avaliação específico para SM (Albuquerque et al., 2009) contemplando aspectos estruturais e funcionais do sistema estomatognático, sendo avaliados por meio do toque, observação da face, movimentação de olhos, lábios, ATM, mandíbula, bochechas, mentoniano, arcada dentaria, língua, palato, palato mole, funções neurovegetativas, mastigação, deglutição, respiração e fala.

$\mathrm{Na}$ terceira etapa, realizou-se a fonoterapia convencional associada à Eletroestimulação Neuromuscular (EENM). As sessões fonoterápicas foram realizadas duas vezes por semana até totalizar doze (12) sessões. No início do tratamento, foi explicado à paciente as sensações esperadas com a aplicação da EENM. Vale ressaltar que a intensidade de cada estímulo foi determinada pelo conforto da paciente, considerando as diferenças individuais sobre dor e desconforto. $\mathrm{O}$ equipamento utilizado para a realização da estimulação foi o da marca HTM Stimulus Physio Maxx com os respectivos cabos de condução, eletrodos que acompanham o equipamento e a caneta de eletroestimulação neuromuscular, que é utilizada especificamente para a estimulação intraoral (região de mucosa) atingindo de maneira pontual os grupos musculares específicos que devem ser trabalhados. A caneta foi adquirida individualmente pela família da paciente.

A Tabela 1 contém os exercícios que foram realizados no decorrer das 12 sessões. 
Tabela 1. Exercícios propostos para as sessões

\begin{tabular}{|c|c|c|}
\hline Sessão & $\begin{array}{c}\text { Exercícios isométricos e isotônicos associados } \\
\text { com a eletroestimulação }\end{array}$ & Exercícios funcionais associados com a eletroestimulação \\
\hline $1^{a}$ Sessão & $\begin{array}{l}\text { Lateralidade de língua. } \\
\text { Sugar língua. } \\
\text { Emitir o fonema /K/ e sílaba /KÃ? } \\
\text { Fechar os lábios tocar um no outro, apertando } \\
\text { como se estivesse passando batom. }\end{array}$ & $\begin{array}{l}\text { Usar as mãos para segurar as bochechas enquanto dá um sorriso bem aberto. } \\
\text { Fechar os lábios e deixar as mãos aplicando força nas bochechas para resistir ao } \\
\text { movimento. } \\
\text { Manter cada posição (sorrindo e não sorrindo) por dez segundos. Percepção do } \\
\text { padrão mastigatório realizado pelo paciente e conscientização do novo padrão a ser } \\
\text { alcançado. } \\
\text { Treinar a mastigação bilateral simultânea. } \\
\text { Orientação para a realização de exercícios domiciliares, mastigação e deglutição } \\
\text { através de alimentos. }\end{array}$ \\
\hline $2^{\mathrm{a}}$ Sessão & $\begin{array}{l}\text { Girar língua. } \\
\text { Tocar com a ponta da língua o palato duro e } \\
\text { tentar tocar com a ponta da língua palato mole }\end{array}$ & $\begin{array}{l}\text { Abrir a boca e as narinas. } \\
\text { Fazer um movimento de franzir o nariz o máximo possível, ao mesmo tempo, } \\
\text { levantar o lábio superior e ficar nesta posição por dez segundos com a ajuda dos } \\
\text { dedos indicadores segurando as bochechas. } \\
\text { Mastigação e deglutição através de alimentos. }\end{array}$ \\
\hline $3^{\mathrm{a}}$ Sessão & $\begin{array}{l}\text { Fazer bico } \\
\text { Fazer sorriso mostrando os dentes. } \\
\text { Fazer sorriso sem mostrar os dentes. }\end{array}$ & $\begin{array}{l}\text { Com a boca fechada, tentar separar os dentes de baixo e de cima sem perder o } \\
\text { contato de um lábio com o outro. } \\
\text { Levar a mandíbula para a frente o máximo possível e ficar nesta posição por cinco } \\
\text { segundos. } \\
\text { Voltar à posição inicial lentamente e repetir dez vezes. Relaxar a boca e fazer um } \\
\text { longo movimento de beijo. } \\
\text { segundos e fechar a boca apertando os lábios entre si. Relaxar e repetir. } \\
\text { Mastigação e deglutição através de alimentos. }\end{array}$ \\
\hline $4^{\mathrm{a}}$ Sessão* & $\begin{array}{l}\text { Sugar língua. } \\
\text { Girar língua. } \\
\text { Estalos de língua. } \\
\text { Sorriso aberto com os dentes a mostra }\end{array}$ & $\begin{array}{l}\text { Fechar os lábios e deixar as mãos aplicando força nas bochechas para resistir ao } \\
\text { movimento. } \\
\text { Treinar a mastigação bilateral simultânea. } \\
\text { Orientação para a realização de exercícios domiciliares. Mastigação e deglutição } \\
\text { através de alimentos. }\end{array}$ \\
\hline $5^{\text {a }}$ Sessão & $\begin{array}{l}\text { Abertura de boca exagerada emitir a vogal /A/ } \\
\text { Com a boca aberta tocar com a ponta da língua os } \\
\text { molares. } \\
\text { Vibração sonorizada de lábios. }\end{array}$ & $\begin{array}{l}\text { Apoiar o braço em uma mesa e colocar a mão fechada embaixo do queixo, } \\
\text { formando um punho com a mão. Em seguida, empurrar o punho e pressionar o } \\
\text { queixo, mantendo a contração por } 5 \text { segundos e repetir o movimento por } 10 \text { vezes, } \\
\text { sugar líquido espesso com canudo de diâmetro aumentado. } \\
\text { Mastigação e deglutição através de alimentos. }\end{array}$ \\
\hline $6^{\mathrm{a}}$ Sessão & $\begin{array}{l}\text { Sorriso aberto com os dentes a mostra } \\
\text { Alongamento com garrote no lábio inferior } \\
\text { Exercício com som plosivo surdo e sonoro /P/ e } \\
\text { /B/ com ajuda e apoio da caneta de } \\
\text { eletroestimulação }\end{array}$ & $\begin{array}{l}\text { Com ajuda das mãos na cabeça, empurrá-la para baixo, até o queixo encostar no } \\
\text { osso da escapula, depois sentido contrário, tentar encostar a nuca nas costas. Repetir } \\
\text { o movimento por } 10 \text { vezes. } \\
\text { Mastigação e deglutição através de alimentos }\end{array}$ \\
\hline $7^{\mathrm{a}}$ Sessão* & $\begin{array}{l}\text { Estalos de língua. } \\
\text { Morder lábio inferior } \\
\text { Protrusão de lábios fechados (bico) com ajuda } \\
\text { dos dedos indicadores e ou com as canetas de } \\
\text { eletroestimulação. }\end{array}$ & $\begin{array}{l}\text { Relaxar a boca e fazer um longo movimento de beijo. Manter por alguns segundos e } \\
\text { fechar a boca apertando os lábios entre si. Relaxar e repetir. } \\
\text { Abrir a boca e as narinas. Fazer um movimento de franzir o nariz o máximo } \\
\text { possível. Ao mesmo tempo, levantar o lábio superior e ficar nesta posição por dez } \\
\text { segundos com a ajuda dos dedos indicadores segurando as bochechas } \\
\text { Mastigação e deglutição através de alimentos. }\end{array}$ \\
\hline $8^{\text {a }}$ Sessão* & $\begin{array}{l}\text { Vibração de lábios sonorizada } \\
\text { Sucção de língua no palato } \\
\text { Abrir e fechar a boca exageradamente } \\
\text { nomear as vogais exageradamente. } \\
\text { A, E, I, O e U. }\end{array}$ & $\begin{array}{l}\text { Dizer "oooo" com os lábios exageradamente arredondados e depois diga "iiiii" } \\
\text { exagerando também no movimento da boca. } \\
\text { Repetir dez vezes estes movimentos alternados, relaxar e realizá-los novamente. } \\
\text { Três séries de dez é o ideal. } \\
\text { Mastigação e deglutição através de alimentos }\end{array}$ \\
\hline $9^{\text {a }}$ Sessão & $\begin{array}{l}\text { Solicitar ao paciente morder suavemente o lábio } \\
\text { inferior com os dentes da arcada superior, onde o } \\
\text { paciente deverá segurar e manter o lábio inferior } \\
\text { Na presença de contração do músculo mentual, } \\
\text { realizar massagens no mento durante o exercício, } \\
\text { associado com o apoio da caneta de } \\
\text { eletroestimulação }\end{array}$ & $\begin{array}{l}\text { Usar as palmas das mãos para criar resistência na testa. Posicionar as palmas das } \\
\text { mãos na lateral da testa, com o final da mão em cima das sobrancelhas. } \\
\text { Alternar expressões de surpresa e raiva com a ajuda das mãos dez vezes. } \\
\text { Depois, fazer o movimento de surpresa e ficar assim por trinta segundos. } \\
\text { Fazer o mesmo com o movimento para baixo e ficar nesta posição por mais trinta } \\
\text { segundos. } \\
\text { Para terminar, repetir os movimentos alternados por dez vezes. } \\
\text { Mastigação e deglutição através de alimentos. }\end{array}$ \\
\hline $10^{\mathrm{a}}$ Sessão & $\begin{array}{l}\text { Aplicar contra resistência com o abaixador de } \\
\text { língua nas regiões superior e laterais da língua; } \\
\text { Emitir o som do fonema /M/ mastigando } \\
\text { Nomear as vogais exageradamente. } \\
\text { A, E, I, O e U, associado com o apoio da caneta } \\
\text { de eletroestimulação. }\end{array}$ & $\begin{array}{l}\text { Colocar os seus dedos indicadores acima das sobrancelhas e fazer movimentos para } \\
\text { baixo enquanto tenta levantar as sobrancelhas. } \\
\text { Repetir este movimento dez vezes para dar mais firmeza à testa e diminuir as linhas } \\
\text { de expressão. } \\
\text { Posicionar os dedos indicadores e médios em forma de V no começo e no final de } \\
\text { suas sobrancelhas. } \\
\text { Puxar a pele para cima e para baixo, pausadamente, por dez vezes. Fazer três séries } \\
\text { de dez movimentos, descansar um minuto e fazer mais três séries de dez. } \\
\text { Mastigação e deglutição através de alimentos. }\end{array}$ \\
\hline $11^{\mathrm{a}}$ Sessão & $\begin{array}{l}\text { Com a boca aberta: O paciente deve posicionar a } \\
\text { água no centro da língua, apoiar sua parte } \\
\text { anterior nas papilas palatinas e, com a boca } \\
\text { aberta, apertá-la contra o palato realizando } \\
\text { movimentos ondulatórios de frente para trás para } \\
\text { levar a água até a faringe. Esse movimento só é } \\
\text { conseguido por meio da elevação do osso hioide, }\end{array}$ & $\begin{array}{l}\text { Apertar os lábios e movê-los para baixo para que os músculos do rosto fiquem } \\
\text { comprimidos. Puxar os lábios para um lado e piscar um olho por um segundo. } \\
\text { Repetir por dez vezes e mudar de lado os lábios e piscar o outro olho. } \\
\text { Fazer três séries de dez movimentos para cada olho, descansar alguns segundos e } \\
\text { fazer mais três séries de dez. } \\
\text { Treinar a função da mandíbula, lábios e língua durante a deglutição de alimentos } \\
\text { sólidos e líquidos. }\end{array}$ \\
\hline
\end{tabular}


por isso é necessário posicionar a mão do paciente debaixo da mandíbula para que perceba essa elevação. Realizamos essa primeira etapa com a boca aberta para estarmos seguros da posição da língua.

\section{$12^{\mathrm{a}}$ Sessão* $^{*} \quad$ Inspire e expire lentamente durante alguns} segundos.

Inspire lentamente, faça uma pausa de alguns segundos e expire lentamente.

Inspire rapidamente e expire lentamente.

s... contínuo; s... pausado; s.... glissando; s.. estacato (pode fazer com $\mathrm{f}, \mathrm{x}$ )

Z... contínuo; $\mathrm{Z}$... pausado; $\mathrm{z}$... glissando; $\mathrm{z}$. .. estacato (pode fazer com $v, \mathrm{j}$ )
Percepção do padrão de fala realizado pelo paciente e conscientização do padrão correto. Treino da fala (fonético).

Orientação para a realização domiciliar de estratégias de: estimulação da sensibilidade; exercícios de tonicidade; monitoramento das funções de respiração, mastigação e deglutição; treino da fala (fonético).

Mastigacão e degluticão através de alimentos.

Exercício do garrote: Esse exercício é realizado para a estimulação do tônus do músculo masseter. Utiliza-se um garrote de $15 \mathrm{~cm}$ de comprimento para morder, este é colocado no lado direito sobre os molares e depois no lado esquerdo. Deve-se morder de 15 a 20 vezes de cada lado.

Posicionar os dedos indicadores e médios em forma de $\mathrm{V}$ no começo e no final de suas sobrancelhas.

Puxar a pele para cima e para baixo, pausadamente, por dez vezes. Fazer três séries de dez movimentos, descansar um minuto e fazer mais três séries de dez. Mastigação e deglutição através de alimentos.

* Sessões em que a paciente faltou. Fonte: Autores (2021).

Neste sentido, é válido destacar que todos os exercícios realizados foram acompanhados da eletroestimulação. Na Tabela 2 evidenciam-se quais foram os parâmetros utilizados para a aplicação na paciente, visando os resultados mais satisfatórios.

Tabela 2. Parâmetros da corrente FES.

\begin{tabular}{|c|c|c|c|c|c|c|c|c|c|c|c|c|}
\hline Sessão & $1^{a}$ & $2^{a}$ & $3^{\mathbf{a}}$ & $4^{a}$ & $5^{a}$ & $6^{a}$ & $7^{\mathrm{a}}$ & $8^{a}$ & $9^{a}$ & $10^{\mathrm{a}}$ & $11^{\mathrm{a}}$ & $12^{\mathrm{a}}$ \\
\hline Frequência & $10 \mathrm{~Hz}$ & $10 \mathrm{~Hz}$ & $10 \mathrm{~Hz}$ & $10 \mathrm{~Hz}$ & $15 \mathrm{~Hz}$ & $15 \mathrm{~Hz}$ & $25 \mathrm{~Hz}$ & $25 \mathrm{~Hz}$ & $25 \mathrm{~Hz}$ & $30 \mathrm{~Hz}$ & $35 \mathrm{~Hz}$ & $35 \mathrm{~Hz}$ \\
\hline Pulso & 150 us & 150 us & 150 us & 150us & 200 us & 200 us & 250 us & 250 us & 250 us & 280 us & 250 us & 250us \\
\hline Rise & $02 \mathrm{~s}$. & $02 \mathrm{~s}$. & $02 \mathrm{~s}$. & $02 \mathrm{~s}$. & $05 \mathrm{~s}$. & $05 \mathrm{~s}$. & $05 \mathrm{~s}$. & $05 \mathrm{~s}$. & $05 \mathrm{~s}$. & $05 \mathrm{~s}$. & $03 \mathrm{~s}$ & 03s. \\
\hline TON & $05 \mathrm{~s}$. & $05 \mathrm{~s}$. & $05 \mathrm{~s}$. & $05 \mathrm{~s}$. & $10 \mathrm{~s}$. & $10 \mathrm{~s}$. & $10 \mathrm{~s}$. & $10 \mathrm{~s}$. & $10 \mathrm{~s}$. & $10 \mathrm{~s}$. & $10 \mathrm{~s}$. & $10 \mathrm{~s}$. \\
\hline Decay & $02 \mathrm{~s}$. & $02 \mathrm{~s}$. & $02 \mathrm{~s}$. & $02 \mathrm{~s}$. & $05 \mathrm{~s}$ & $05 \mathrm{~s}$. & $05 \mathrm{~s}$. & $05 \mathrm{~s}$ & $05 \mathrm{~s}$. & 03s. & 03s. & 03s. \\
\hline TOFF & $15 \mathrm{~s}$. & $15 \mathrm{~s}$. & $15 \mathrm{~s}$. & $15 \mathrm{~s}$. & $10 \mathrm{~s}$. & $10 \mathrm{~s}$. & 08s. & 08s. & 08s. & $10 \mathrm{~s}$. & $05 \mathrm{~s}$. & $05 \mathrm{~s}$. \\
\hline Intensidade & Baixa & Baixa & Baixa & - & Baixa & Baixa & Média & Média & Média & Média & Média & Média \\
\hline Tempo & $10 \mathrm{~m}$ & 10. & $10 \mathrm{~m}$ & - & $12 \mathrm{~m}$. & $12 \mathrm{~m}$ & $15 \mathrm{~m}$ & $15 \mathrm{~m}$. & $15 \mathrm{~m}$. & $15 \mathrm{~m}$ & $15 \mathrm{~m}$ & $15 \mathrm{~m}$. \\
\hline
\end{tabular}

Fonte: Autores (2021).

A Figura 1 exemplifica como ocorreu a eletroestimulação, os pontos de disposição dos eletrodos na criança, sendo aplicada no músculo bucinador, risório e masseter no lado paralisado e músculo digástrico.

Figura 1. Paciente realizando a eletroestimulação

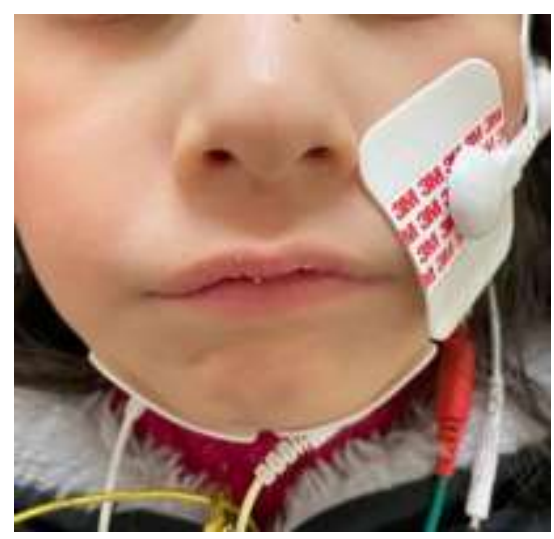

Fonte: Arquivo Pessoal (2021).

Por fim, a quarta e última etapa consistiu em uma nova avalição clínica, objetiva e subjetiva da SM, da mesma forma que a avaliação inicial, para análise comparativa dos resultados e avaliação da eficácia da terapêutica. 


\section{Resultados e Discussão}

O caso analisado foi de uma paciente do sexo feminino, 6 anos de idade, com diagnóstico clínico da Síndrome de Moebius, cuja principal queixa relatada pela responsável foi a dificuldade na fala da criança. Conforme anamnese realizada, por intermédio de ficha com avaliação estrutural e funcional dos órgãos fonoarticulatórios, realizada por um profissional fonoaudiólogo, obtiveram-se algumas percepções que se mantiveram, e outras que se alteraram, no decorrer do tratamento.

O planejamento terapêutico teve como objetivo geral estimular a musculatura paralisada em decorrência da SM, com foco no desenvolvimento da mobilidade dos órgãos fonoarticulatórios e funções estomatognáticas: mastigação, deglutição, sucção, respiração e fala, assim também estimulou-se a expressão facial por meio do trabalho com os grupos musculares responsáveis pela expressividade da face.

$\mathrm{Na}$ avaliação, inicialmente observou-se acerca da movimentação da cabeça a realização de todos os movimentos solicitados, tais como a rotação para os lados direito, esquerdo bem como para frente e para trás, não havendo nenhuma complicação. A face da criança demonstrou assimetria entre as hemifaces esquerda e direita, e padrão facial braquifacial. Já no critério de movimentação dos olhos, a criança não apresentou nenhuma alteração e/ou diferença que fosse potencialmente relevante e que interferisse no tratamento da SM. Tais características se mantiveram ao final do tratamento.

Já na avaliação dos órgãos do sistema estomatognático, quanto aos lábios, no início do tratamento, eram abertos, apresentando hipotonia e hipofuncionais, o freio labial é normal com mobilidade alterada de língua (Figura 2).

Observou-se uma transformação relativa ao sorriso da paciente (Figura 3), podendo ser comparada ao início do tratamento (Figura 2). Foi notável a melhora na movimentação labial da criança, que ao início da terapia fonoaudiológica não realizava movimento para sorrir, já nas últimas sessões apresentava perceptível evolução na movimentação de comissura labial no lado paralisado.

Figura 2. Sorriso ao início do tratamento.

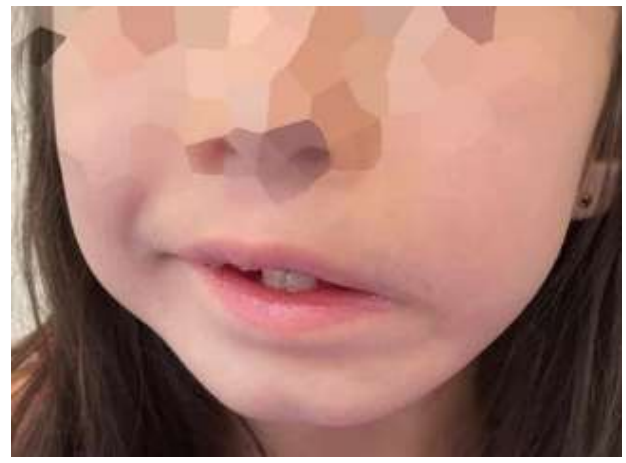

Fonte: Arquivo Pessoal (2021).
Figura 3. Sorriso ao final do tratamento.

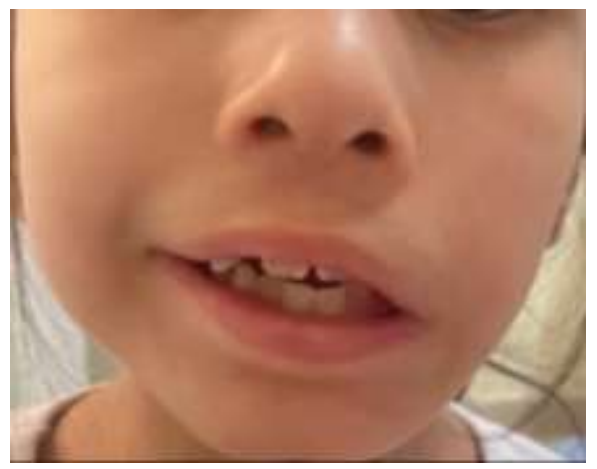

Fonte: Arquivo Pessoal (2021).

Em relação às praxias orofaciais, observou-se algumas dificuldades, com movimentos restritos, dos quais os principais foram abrir a fechar a boca e levá-la para a direita, sendo perceptível que sua mobilidade era alterada. Após as sessões fonoaudiológicas, do protocolo combinado com a utilização da corrente FES, percebeu-se que algumas praxias foram paulatinamente sendo adquiridas pela paciente, sendo que ao final tratamento proposto, a mobilidade já era considerada como normal, realizando todos os exercícios propostos, tais como fechar e abrir a boca contra resistência, levá-la para a direita, esquerda, para trás, para frente, bem como realizar movimentos circulares.

As bochechas apresentavam hipotonia e os lábios sem vedamento (Figura 4). Mesmo frente ao tratamento proposto não foram identificadas evoluções perceptíveis nas bochechas, mas, com uma melhora evidente no vedamento labial (Figura 5). 
Figura 4. Lábios abertos e bochechas hipotônicas.

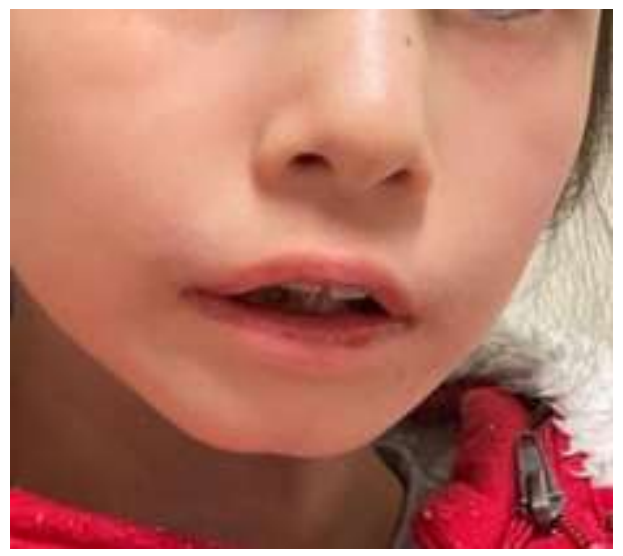

Fonte: Arquivo Pessoal (2021).
Figura 5. Evolução da paciente.

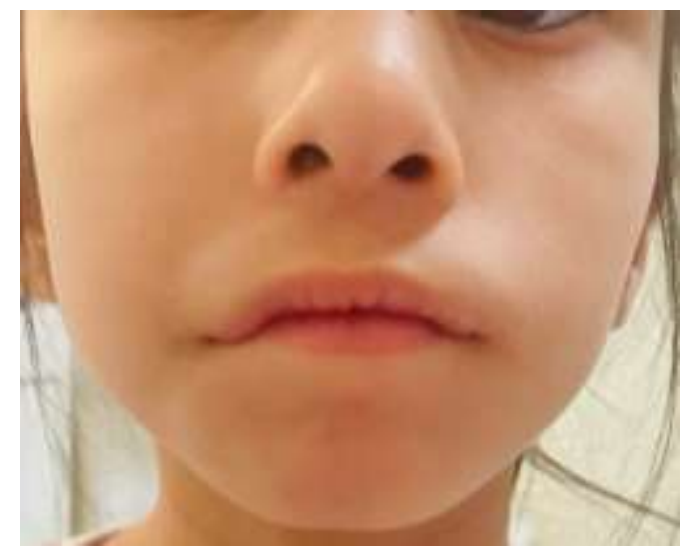

Fonte: Arquivo Pessoal (2021).

Observa-se que a melhora no vedamento labial e a respiração, que passou do modo oral para misto, auxiliaram na diminuição do ressecamento dos lábios e em seu aspecto estético. Além disso, no início do tratamento, acerca das praxias orofaciais, quanto a língua, a paciente fazia apenas os movimentos para dentro e para fora, estalar, lateralizar, para cima e para baixo dentro e fora da boca. No fim das sessões, foram agregados à paciente o movimento de afinar e alargar, sendo notável uma pequena e gradual evolução.

A arcada dentária com dentição mista, haja posto a idade da criança, com apinhamentos e diastema, apresentando uma arcada alterada, classe II, possuindo um desvio para a esquerda em sua linha média. Relacionado à língua, percebeu-se que sua tonicidade era hipotônica, cujo aspecto era fasciculado com desvio curto e mobilidade alterada. Ao findar das sessões, a mobilidade da língua da paciente já era normal, pois estava sendo estimulada no decorrer da terapia fonoaudiológica, possibilitando esse avanço. De acordo com Matos et al. (2020) a estimulação do paciente com SM, desde a mais tenra idade, contribui positivamente com o seu desenvolvimento, possibilitando maior qualidade de vida, ao seguir protocolos específicos que contribuam à sua gradativa melhora. No que se refere ao palato duro é ogival e o palato mole com movimentação diminuída no início da terapia. Ao fim do tratamento, percebeu-se um avanço pois o palato mole apresentou movimentação normal.

Para a realização da avaliação das funções estomatognáticas, verificou-se que a sucção para líquidos era eficiente, sem escape, sem canolamento de língua e com ritmo. Para líquidos pastosos finos e grossos não havia canolamento, nem participação de bucinador, apresentando ritmo regular.

Inicialmente, ao avaliar-se a mastigação e deglutição, foram utilizados três alimentos distintos: pão francês, waffer e pão de queijo. Observou-se, que no início do tratamento a criança apresentava mastigação alterada, com movimentos amplos e imprecisos para os alimentos: pão francês e pão de queijo, e movimentos mais coordenados no waffer, que exige menor atividade muscular, com incisão anterior e lateral, mastigação unilateral, movimentos verticalizados e sem contração de masseter, sendo necessário empurrar alguns pedaços de alimento com o dedo, sem vedamento labial.

$\mathrm{Na}$ análise posterior às sessões, a paciente já conseguia realizar movimentos rotatórios de mastigação, de forma bilateral alternada, formando bolo coeso e centralizando-o na língua, além de apresentar velocidade de mastigação normal. Ao final do tratamento a criança já não utilizava o dedo para introduzir o alimento na cavidade oral. Finalmente, a respeito da respiração a análise demonstrou que inicialmente ela possuía respiração do tipo mista, de modo oral e silenciosa. Ao fim do tratamento, era mista, de modo também misto e silenciosa.

Pinheiro et al. (2018) evidencia que a eletroestimulação colabora de forma muito positiva para o desenvolvimento da mobilidade da língua dos indivíduos, pois atua diretamente na musculatura bem como na respiração, fazendo com que haja 
uma transformação no comportamento labial no processo de deglutir e de mastigar, expressando, portanto, as possibilidades de evolução a partir dessa terapêutica, fato que também foi observado no caso em questão, com as evoluções funcionais na mastigação, deglutição e respiração.

Percebeu-se, por meio das análises realizadas do protocolo, bem como o acompanhamento da paciente nas sessões, que os resultados da terapia juntamente com a eletroestimulação FES apresentou resultados positivos, como pode ser percebido no avanço em relação às práticas orofaciais, nos progressos relacionados à mastigação e deglutição, além da sensibilidade da região paralisada.

Destaca-se que a falta de comprometimento por parte da família em possibilitar o acesso da criança às sessões de fonoaudiologia também dificultaram o processo e o protocolo. Sabe-se que principalmente relacionado às crianças, o trabalho interdisciplinar e a participação da família é fundamental. Fernandes e Souto (2021), Simoni et al., (2019) e Souza e Lopes (2015) esclarecem que a participação da família para o tratamento fonoaudiológico é imprescindível, sobretudo na contribuição para as evoluções no tratamento, haja posto os estigmas, o comprometimento da autoestima do indivíduo e as dificuldades emocionais e em relação à socialização. Contribuir para que os pais e/ou responsáveis compreendam o tratamento fonoaudiológico como uma etapa importante para o desenvolvimento do paciente, é o primeiro passo para o estabelecimento de um tratamento efetivo, salvaguardando o envolvimento deste ao tratamento, ao passar para a criança mais confiança, afeto e segurança.

A inserção de um plano de intervenção interdisciplinar aos familiares é uma das possibilidades que o serviço de saúde pode viabilizar, para que as necessidades sejam adequadas e para que a participação seja eficaz, sendo imprescindível o comprometimento familiar para o pleno desenvolvimento e obtenção dos melhores resultados possíveis no tratamento, além da mudança de hábitos que visem o cuidado com a criança e com suas necessidades (Fernandes \& Souto, 2021).

No caso da SM, uma alteração cujo tratamento demanda um relativo esforço por parte do paciente e do profissional, os exercícios domiciliares, para além das sessões, são essenciais (Souza \& Lopes, 2015). Notou-se, a partir de diálogos com a criança e com a própria família, que os exercícios solicitados não eram realizados em ambiente externo ao hospitalar, o que evidencia que o tratamento ocorria exclusivamente com a presença da fonoaudióloga.

Percebe-se que a escassez de estudos que discorram sobre a SM trata-se de uma problemática para a efetivação de práticas que possibilitassem o avanço da paciente em relação às dificuldades. Tal falta, reflete em prejuízos psicossociais aos indivíduos portadores desta doença, uma vez que compromete a qualidade de vida deles, pois a sociedade não possui informações acessíveis e atualizadas sobre o assunto, trazendo rótulos e estigmas prejudiciais ao desenvolvimento integral dos pacientes. Sobretudo no caso das crianças, a impossibilidade e a dificuldade na articulação da fala, traz consequências ao convívio social em fase escolar, à aprendizagem e até mesmo à autoestima (Goulart \& Chiari, 2007; Alves et al., 2015).

O estudo de Alves et al. (2015) explora que as principais queixas relatadas pelos pacientes diagnosticados com a SM, referem-se à fala, sendo esta síndrome dificultosa para o indivíduo, pois traz dificuldades na mastigação, na comunicação, e afetam a vida social dos pacientes, haja posto o atraso no desenvolvimento destes aspectos. Em contrapartida, para a EscodaFrancolí, Sánchez-Garcés e Gay-Escoda (2009) as principais alterações pertinentes à SM, são orais, tais como a micrognatia, fissura lábio palatina (FLP), musculatura diferenciada e alterada, dentição hipoplásica e dificuldade no controle da salivação, que consequentemente traz dificuldades na oralidade do indivíduo, ou seja, em sua comunicação. Albuquerque et al. (2009) apontam a hipernasalidade como um dos principais prejuízos na articulação fonética.

Ressalta-se que, embora o tratamento fonoaudiológico tenha demandado maior tempo em sua realização, haja posto a ausência da paciente em algumas sessões por motivos pessoais, obtiveram-se resultados positivos, confirmando a hipótese inicial da efetividade prática da eletroestimulação atrelada à terapia de fonoaudiologia convencional. Os benefícios foram 
perceptíveis não apenas esteticamente, mas sim na qualidade de vida da criança, nas funções orofaciais e na constante evolução da sensibilidade orofacial da paciente e de sua própria autoestima.

\section{Conclusão}

Conclui-se que a eletroestimulação associada à terapia fonoaudiológica apresentou resultados positivos, principalmente no que tange às funções orofaciais, mastigação, deglutição, sucção e respiração, sendo esta uma proposta de tratamento viável para pessoas acometidas pela SM. A perspectiva para o desenvolvimento de novos estudos, evidenciam a importância de atrelar novas técnicas à fonoterapia convencional, com o intuito de trazer para a realidade novas possibilidades de trabalho para o tratamento de pacientes que são acometidos pela SM, bem como por outras síndromes, cujos impactos são perceptíveis.

Contudo, a escassez de materiais acerca da temática ainda é evidente, sendo necessário que os pesquisadores deem continuidade aos estudos sobre a SM e suas possibilidades terapêuticas. A SM é uma alteração, cuja comunidade científica ainda busca estudar e trazer contribuições, no intuito de encontrar possibilidades para o seu tratamento, com a finalidade de proporcionar aos indivíduos que a detém, qualidade de vida, sem que suas atividades sejam severamente comprometidas. O tratamento fonoaudiológico é de suma importância, pois viabiliza transformações que contribuem para o desenvolvimento do indivíduo, sobretudo no que tange à sua fala e a expressão facial. Percebe-se que a atuação interdisciplinar articulada com a família é de grande importância e quando a terapia é levada a sério, os resultados positivos são garantidos.

\section{Referências}

Albuquerque, T. C. A. L., Barreto, R. R. S., Costa, T. C. M., \& Guedes, Z. C. F. (2009). Sequência de Möbius: protocolo de anamnese e avaliação - relato de caso. Revista da Sociedade Brasileira de Fonoaudiologia, 14(1), 115-122.

Alves, G. A. S., Lima, I. L. B., Lima, J. A. S., Lucena, B. T. L., \& Delgado, I. C. (2015). Alterações de fala em crianças com Síndrome de Moebius: análise da literatura. Revista do GEL, 12(1), 33-45.

Coelho, V. L. de H. (2012). Abordagem fisioterapêutica na síndrome de moebius. Revista Nova fisio. https://www.novafisio.com.br/abordagemfisioterapeutica-na-sindrome-de-moebius/.

Diogo, F. S. F., Aguiar, S. M. H. C. A., Faria, C. C. M., \& Rocha, G. S. T. (2009). Utilização do misoprostol (Cytotec®) como droga abortiva e sua relação com a síndrome de moebius: relato de caso. Revista Odontológica de Araçatuba, 30(1), 31-35. https://pesquisa.bvsalud.org/portal/resource/pt/biblio-856848.

Escoda-Francolí, J., Sánchez-Garcés, M. A., \& Gay-Escoda, C. (2009). Oral implant rehabilitation in a patient with Moebius syndrome. Medicina Oral Patologia Oral y Cirugia Bucal, 14(6), 295-298. https://pubmed.ncbi.nlm.nih.gov/19300371/.

Fernandes, D. R., \& Souto, B. G. A. (2021). Participação familiar no cuidado de crianças com transtorno fonológico. Audiology Communication Research, 26(1), 1-10. https://www.scielo.br/j/acr/a/pcn5F7w87vFL4xYYMyCZmVL/.

Freitas, A. C., Nelson-Filho, P., Queiroz, A. M., Assed, S., \& Silva, F. W. G. P. (2006). Síndrome de moebius: relato de caso clínico. Revista de Odontologia

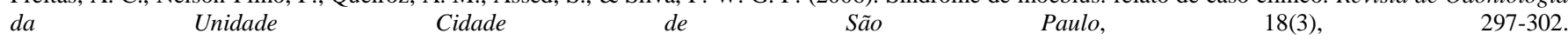
https://arquivos.cruzeirodosuleducacional.edu.br/principal/old/revista_odontologia/pdf/setembro_dezembro_2006/sindrome_moebios.pdf.

González C. H., Marques-Dias M. J., \& Kim C. A. (1998). Congenital abnormalities in Brazilian children associated with misoprostol misuse in first trimester of pregnancy. Lancet, 351: 1624-1627.

Goulart, B. N., \& Chiari, B. M. (2007). Prevalência de desordens de fala em escolares e fatores associados. Revista de Saúde Pública, 41(5), 726-731.

Kumar D. (1990). Moebius syndrome. J Med Genet, 27(1), 122-126. https://www.ncbi.nlm.nih.gov/pmc/articles/PMC1016933/pdf/jmedgene00040-0050.pdf.

Lammens M., Moerman P., \& Fryns J. P. (1998). Neuropathological findings in Moebius syndrome. Clin Genet 54(1), 136-141. https://pubmed.ncbi.nlm.nih.gov/9761392/.

Larrandaburu M., Schüler L., Ehers J.A., Reis A. M., \& Silveira E. L. (1990). The occurrence of Poland and Poland-Moebius syndromes in the same family. Clin Dysmorphol, 8(2), 93-99. https://pubmed.ncbi.nlm.nih.gov/10319197/.

Lima, N. M. F. V., \& Cunha, A E. R. L. (2011). Efeito da eletroterapia na paralisia facial de Bell: Revisão de literatura. Ensaios e ciência: ciências biológicas, agrárias e da saúde, 15(3), 173-182.

Lipson, T., Webster, W., \& Weaver, D. D. (1990). The Moebius syndrome: aetiology, incidence of mental retardation, and genetics. J Med Genet 27 (1), 533 534. 
Research, Society and Development, v. 11, n. 1, e6311124310, 2022

(CC BY 4.0) | ISSN 2525-3409 | DOI: http://dx.doi.org/10.33448/rsd-v11i1.24310

Martí-Herrero M., Cabrera-López J. C., Toledo L., Pérez-Candela V., \& Bon- net D. (1998). Síndrome de Moebius. Tres formas diferentes de presentación. Revista Neurología, 27(1), 975-978

Miller M. T., \& Strömland K. (1999). The Möbius sequence: a relook. J AAPOS 3(1), 199-208.

Netter, F. H. (2015). Atlas de anatomia humana. Elsevier.

Pinheiro, D. L. S. A., Alves, G. A. S., Fausto, F. M. M., Pessoa, L. S. F., Silva, L. S., Pereira, S. M. F., \& Almeida, L. N. A. (2018). Efeitos da eletroestimulação associada a treino mastigatório em pessoas com síndrome de down. CoDAS, 30(3), 1-20. https://www.scielo.br/j/codas/a/8sPTQPGd5GjyDxpLNGjKqjz/abstract/?lang=pt

Pupo Filho, R. A., Cardoso, T. A. L., Martins, J. R. O., Micheletti, J. A., Carvalho, J. F., Moraes, M. G., \& Duarte, M. S. N. (1999). Síndrome de Moebius: uma patologia emergente no Brasil / Moebius syndrome in Brazil. Revista Paulista de Pediatria, 17(2), 91-94. http://portal.revistas.bvs.br/index.php?search=Rev.\%20paul.\%20pediatr\&connector=ET\&lang=pt.

Romansina, D., Simões-Zenari, M., \& Nemr, K. (2020). Eletroestimulação funcional associada à fonação em mulheres sem alterações vocais. CoDAS. 33(1), 1-7. https://www.scielo.br/j/codas/a/nx4n57tjdZmbqDKPsD6FbCN/?format=pdf\&lang=pt.

Simoni, S. N., Leidow, I. C., Britz, D. L., Moraes, D. A. O., \& Keske-Soares, M. (2019) Impacto dos distúrbios dos sons da fala: a percepção da família e da criança. Revista CEFAC, 21(3), 1-13. https://www.scielo.br/j/rcefac/a/pCptq3GZns6zSTcg7SDxT9J/?format=pdf\&lang=pt.

Souza, D. M. B., \& Lopes, S. M. B. (2015). Percepção da família em relação à atuação fonoaudiológica em um ambulatório. Revista CEFAC, 17(1), 80-87. https://www.scielo.br/j/rcefac/a/PdVBnyQS6BfWykmf5mrCCdy/?format=pdf\&lang=pt. 